\title{
EDUCATIONAL LABORATORY FOR THE STUDY OF POWER CONVERTER THROUGH A WEB BROWSER
}

\author{
Luis Eguizabal, Alfonso Lago, Luis Rodriguez, Carlos Peñalver, Andres \\ Nogueiras \\ UNIVERSIDAD DE VIGO. Escuela Universitaria de Ingenieria Técnica Industrial. C/ \\ Torrecedeira n 86. 36208 Vigo, Spain. Tel.: +0034986813962 Fax: +0034986813644
}

\begin{abstract}
The solution that is shown in this paper allows to students of different subject of Power Electronics, the accomplishment of a complete study of $\mathrm{DC} / \mathrm{DC}$ converters, by means of two different exercises:

- Design. The students must evaluate the behavior of the power circuit in open loop, choosing among different values of components for the implement of the studied topology. The software facilities tools for the measure of the main waveforms of the topology, such as: inductor current, control signal to the switch, output voltage...

- Test. After the study of the power circuit, the software allows to analyze response of the power converter in close loop, by means of a set of test: line regulation, load regulation...

The developed system is based on a test bench manufactured by HewlettPackard. Taking this one as starting point, a software application has been developed using the graphical programming language "LabVIEW". This programming tool combined with CGI (Common Gateway Interface), allows the remotely execution using a web browser, increases the versatility and utility of the system.
\end{abstract}

Keywords: Virtual laboratory, Power converter, LabVIEW programming, Elearning, CGI programming. 


\section{INTRODUCTION}

Nowadays, with the generalization of the use of PC's (Personal Computer) in education, new tools have appeared that are very helpful in improving the classic methods of education.

Among these new education tools developed around the use of PC's, it is possible to emphasize:

1. The use of simulation software, which allows multiple kinds of analysis in the study of circuits, thus obtaining results that in any other way would be very difficult to obtain.

2. The possibility of improving the presentation methods, as much of the theoretical classes as the practical ones.

3. New study techniques of circuits in the practical classes, based on the connection of programmable instrumentation equipment to a PC, by means of an instrumentation bus.

4. The use of Internet, to facilitate the access to the information available in the network, and also giving the possibility of making practical exercises at a distance, which has come to be known as "Remote Experimentation".

This article presents an educational laboratory, which tries to explore the possibilities shown in 3 and 4 .

The increasing interest in remote experimentation has produced many studies in this field, among which it is possible to mention:

- A real time system for the control of robot arms is described in [1]. In this article a remote system based on LabVIEW, is used the government of circuits for engine control. This solution allows to the students to make remote experiments, and the teacher to improve his presentations.

- Also it is interesting to mention, by its relation with this article, the system developed by So and Lee [2], which allows engineering students to control a pilot-scale DC servomotor remotely. The user is able to extract important characteristics of the physical system and tune a PID controller. The control system is based in data acquisition cards.

- The article [3] is focused in the study of a series of transducers connected to $\mathrm{I} / \mathrm{O}$ analogical and digital modules, employed in chemical process control.

The main advantages that contribute this educational laboratory are:

- It allows the study and test of DC/DC converter, without the necessity expensive equipment.

- The software developed is friendly, versatile and allows the carrying out the laboratory exercises without presence.

- The evaluation of the student is done on-line and automatically for the software. 
- Too the educational laboratory allows to introduce to the students in the use of electronic instrumentation.

\section{THE TEST BENCH FOR THE STUDY OF DC-DC CONVERTERS}

The hardware system necessary to implement the virtual laboratory is based on a set of programmable instruments interconnected using GPIB (General Propose Instrumental Bus) [4]. In order to be able to control and supervise this instrumentation, a PC is used. A GPIB card allows to connect the PC and programmable instrumentation. Next, each one of the instruments that form the test bench is described briefly.

a) Supplying and load elements:

- HP667A. 500W 60V/9A Programmable DC Source, protected against overvoltages and overcurrents.

- HP6050A. 300W 60V/60A Electronic Load, it can be programmed in: current, voltage or resistance mode.

b) Measurement instruments and waveform generator:

- HP54602B. 4 channels 150Mhz Digital Oscilloscope.

- HP34401A. Digital Multi-Meter (DMM) 6.5 digits of accuracy.

- Agilent 33220A. Function-Arbitrary Waveform Generator, $20 \mathrm{MHz}$.

c) Configuration and interconnection elements:

- HP75000. VXI bus Data Acquisition Mainframes. This element is used for interconnect the Unit Under Test (UUT) and the instruments. It works like controller of the test bench. The HP7500 has connected in its VXI expansion slots: three $4 * 4$ matrixes of contacts HPE136A cards, and two HPE 1664 cards of 16 relays. With these contacts and relays the definition of the connections between the UUT and the instruments of the test bench is possible.

The relays are used for connecting the instruments and the UUT, and to change the operation mode of the topology. For example, changing between several values of inductances and capacitors of the circuit.

\section{SOFTWARE DESCRIPTION}

The software developed has two main modules:

- Test Software: handle the instruments from the PC using GPIB commands. 
- Network Software: communicates the host and different clients who receive information from the test bench.

\subsection{Control software.}

In order to develop this module, the widely used graphical programming language LabVIEW (Laboratory Virtual Instrument Engineering Workbench), has been used. LabVIEW is a powerful and flexible programming language used for: acquisition and analysis of data, control of instruments [5].

The developed application is located in a personal computer that is connected to the test bench; it allows the execution of educational laboratory in local and remote mode. In this last case, the PC works as a bridge between the test bench and the remote client.

As the instruments are controlled through a GPIB bus, the communication between the host and the instruments is based in text messages. The main part of the software is in charge to generate the necessary messages for the control of the different devices, and to understand the answer messages that are received.

Three Virtual Instruments (VI), basically related to GPIB protocol 488.2, will be used:

- MakeAddr: generates GPIB compound addresses, necessary to control the matrix of contacts and the relays.

- Send: sends a text message to the element whose direction is indicated like parameter.

- Receive: read from the GPIB bus a message of a certain length.

Following the structure of these three virtual instruments, two news VI were created: GPIBcommand and GPIBquery. It is interesting to emphasize the second one, that is compounded of the Send and Receive commands. So that, only one VI is used for request information and then, the bus is scanned until the answer message is received. Therefore, with these two new virtual instruments, and once the addresses of the instruments included in the test bench are known, the control software automatizes the access to the test bench.

Once detailed the software that makes GPIB communication, it will be explained the procedure used for the remote control of the test bench.

\subsection{Remote access application.}

The remote access to the test bench is based on the programming of CGI applications, so the bench can be controlled through of a web browser.

The remote access application contains two modules: 
1. A program developed in LabVIEW for controlling the instruments by means of the GPIB. A virtual instrument of this module must be generated the necessary inputs and outputs for controlling and executing.

2. The second module must link the requests for information from the client to the server. Thus, when in a client computer a button of the designed application is clicked, the effect in the GPIB communication program is the same as this one were being executed in local way.

CGI applications can be written in most of the programming languages, although the most used it is the Perl language. In order to be able to use this communication interface in LabVIEW, the Internet tools for LabVIEW are needed. These tools contain virtual instruments for the CGI communication [6] [7].

The Internet tools of LabVIEW provide a virtual instrument called $G$ Web server. It is the server who uses LabVIEW to be able to connect a VI to Internet.

CGI application consists of three basic parts:

- A virtual instrument that makes the CGI communication between the server and the browser. Its function is to receive the request for information from the server, it sends this request to another virtual instrument that processes it, and finally, it sends the answer to the browser. The reception of this information is made by means of CGI instrument: Read Request.vi, and it is sent by CGI Release.vi.

- The virtual instrument that processes the request for information, have like main missions look for in the Keyed Array with the help of the virtual instrument Keyed Array Index, the necessary inputs, and send them to the virtual instrument that is wanted to control. Finally, it is created the web page to send to the browser.

- The virtual instrument that the clients are going to be able to handle through the browser is the one in charge to communicate with the test bench.

The virtual instruments more important to program this CGI communication are: CGI Read Request.vi, CGI Write Reply.vi, CGI Release.vi, CGI Parse URL-Encoded Param String.vi, and Keyed Array Index.vi.

Therefore, with this software that implements CGI communication, the students can use the system with a simple web navigator, without LabVIEW in their computers. 


\section{THE WEB SITE}

The educational laboratory allows the study of power converters in two ways:

- Design. In this section the students can use the developed system to determine the values of the power components that implement the power circuit, analyzing how these elements affect to the electrical response of the converter. In this phase, the converter works in open loop.

- Test. Once designed the topology, in this second exercise the quality of the converter is studied. For it, working now the circuit in close loop, a test set has been defined: line regulation, load regulation, crossed regulation... Thus it is possible to establish the converter output quality under different situations.

In order to facilitate the access to the study system, a website was developed. Figure 1 shows one of the web pages. In the left side of the window, it is accessible

1. TEORÍA CC/CC (DC-DC theory). The students have available a resume of DC/DC converter theory.

2. Banco de pruebas (Test bench). A description about the test bench is included, where is indicated the main characteristics of the instruments.

3. DISEÑO CC-CC (Power circuit design). This option is explained in the next paragraph.

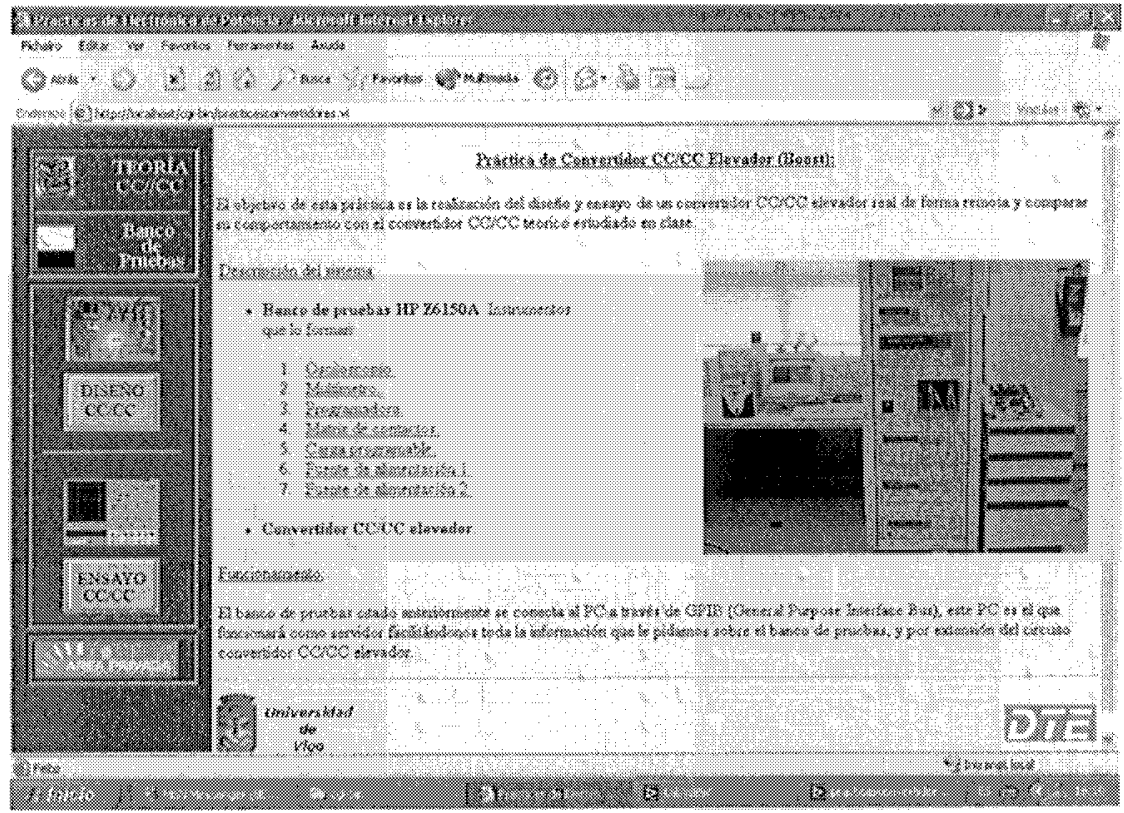

Figure 1. Website for the study of DC/DC converter 
4. ENSAYO CC-CC (Test of the convert). See paragraph "Procedure for the definition and accomplishment of a test sequence".

5. AREA PRIVADA (Private area). In this button, and through of a password, the teacher or the system manager can supervise the configuration options of the website. For example: management of students (accomplishment and evaluation of the exercises), management of the practical exercises.

The educational laboratory evaluates of automatic form to the students, comparing their answers with the results defined by the professor. This process generates a list of qualifications that can be printed or exported to excel. In the figure 2 , is shown a web page where the professor defines the correct range of the results.

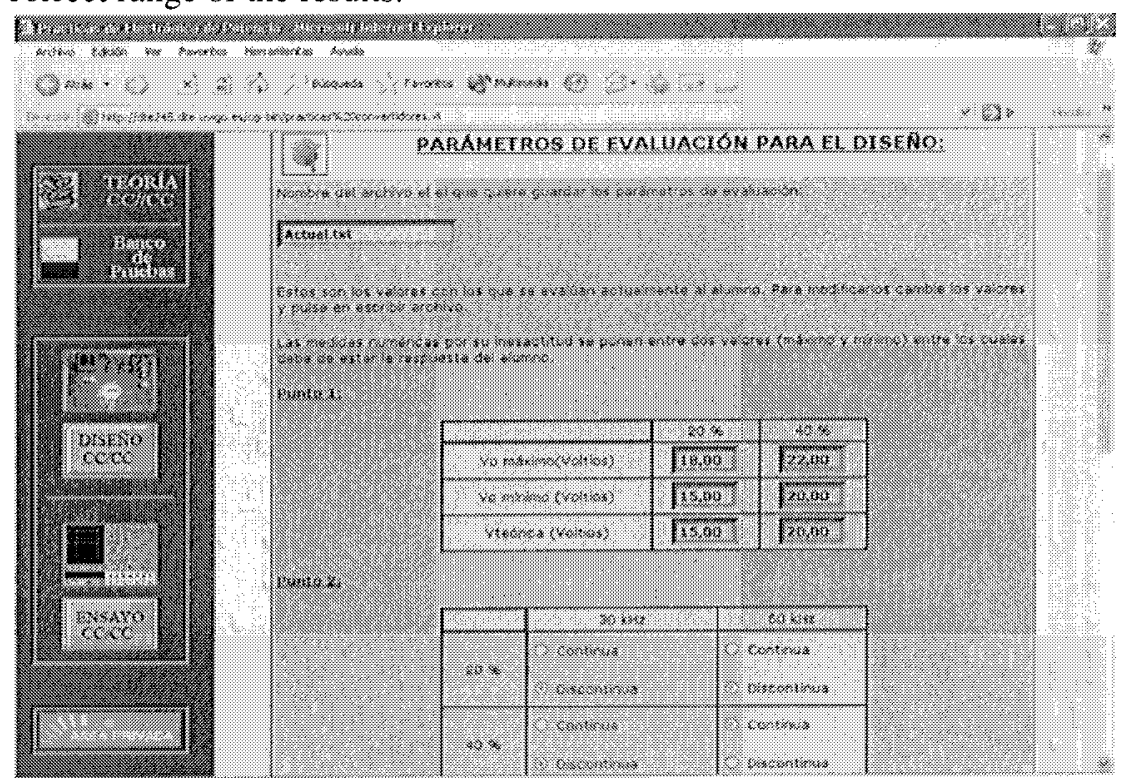

Figure 2. Web page for definition of the correct range of the results

\section{POWER CIRCUIT DESIGN}

As it was indicated in previous sections, the developed system is useful for the design and test of DC/DC converters.

From the design point of view, the software allows to accede to all the instrumentation of the test bench. So, it is possible perform a manual configuration of the bench, choosing the connection and waveform to 
measure. The possibility of changing the connections allows to modify the physical structure of the converter. This option is useful to analyze how the values of the power component affect in the behavior of the topology. The students must accomplish each one of the steps indicated in the report of the practical exercises, in order to analyze the effect of: the: duty cycle (D), the frequency (f), the inductance and capacitor value; on the response of the circuit.

Thus in figure 3 , it can be observed the basic signals of the step-up converter: the upper waveform is the control signal to the switch, the middle represents the inductor current and the lower waveform is the output voltage. For the measurement of these values, the converter works in open loop, and the signal for the switch control is obtained with a function waveform generator.

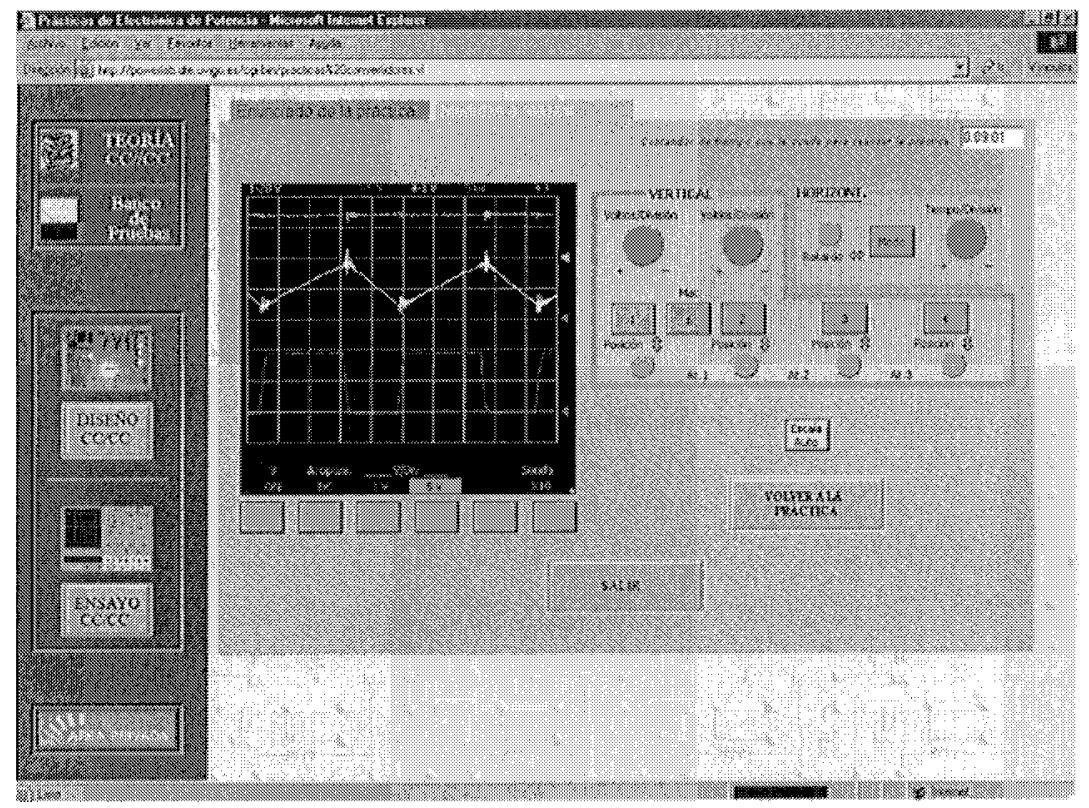

Figure 3. Example of the obtained measurements

\section{PROCEDURE FOR THE DEFINITION AND ACCOMPLISHMENT OF A TEST SEQUENCE}

Once the power circuit is design, the student must test the behavior of the power converter in close loop. In this section is described the procedure for execution of a test sequence. 
The steps to follow for the definition and accomplishment of a new test are:

1. Make physical connections between UUT and the test bench.

2. Information Editor. A name to the converter is provided and the inputs and outputs are defined.

3. Definition of connections. The connections between the UUT and the test bench are defined.

4. Specifications Editor. In this section are introduced the maximum and minimum voltage and currents values, that can handle the converter.

5. Test definition. This section defines the test sequence.

6. Limits and conditions test. In this step the students can define the execution conditions of the each test.

Steps: 1, 2 and 3; are defined by the teacher, the student can only display the configuration values.

As an example, the figure 4 shows one of these configuration windows, in which it is defined the test sequence. At the lower of the windows are available the buttons in order to select the other configuration windows.

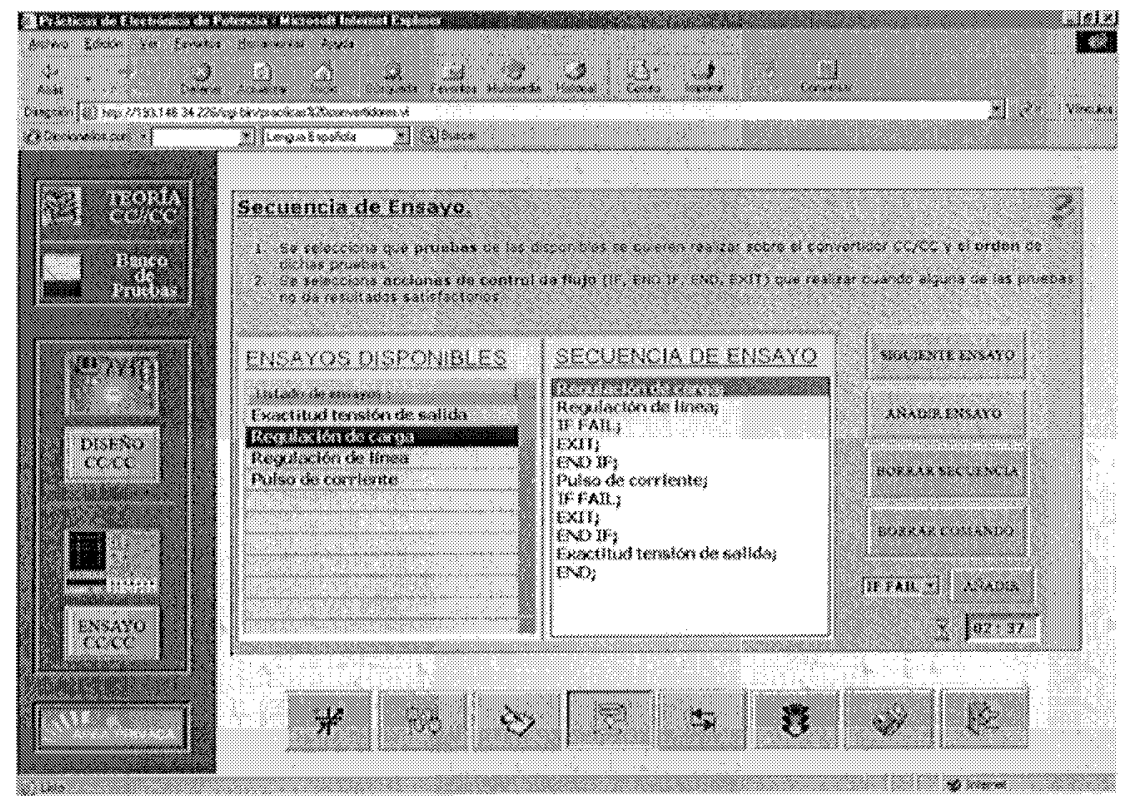

Figure 4. Window for the definition of the test sequence.

Once made these six steps, the system is ready for the execution of the test sequence. For it, the software enables a new window, in which is possible control the tests execution, and to observe the result obtained. 
In this analysis mode also it is possible to visualize waveforms through the instrumentation window. As example, in figure 5 is shown the obtained answer after the execution of an analysis of "current pulse in the load". The current pulse applied can be observed, it varies of $0.4 \mathrm{~A}$ to $2 \mathrm{~A}$., and the variation that experiences the output voltage is only of the order of $20 \mathrm{mV}$. This last measurement is solely shown the $\mathrm{AC}$ component, to obtain a greater definition.

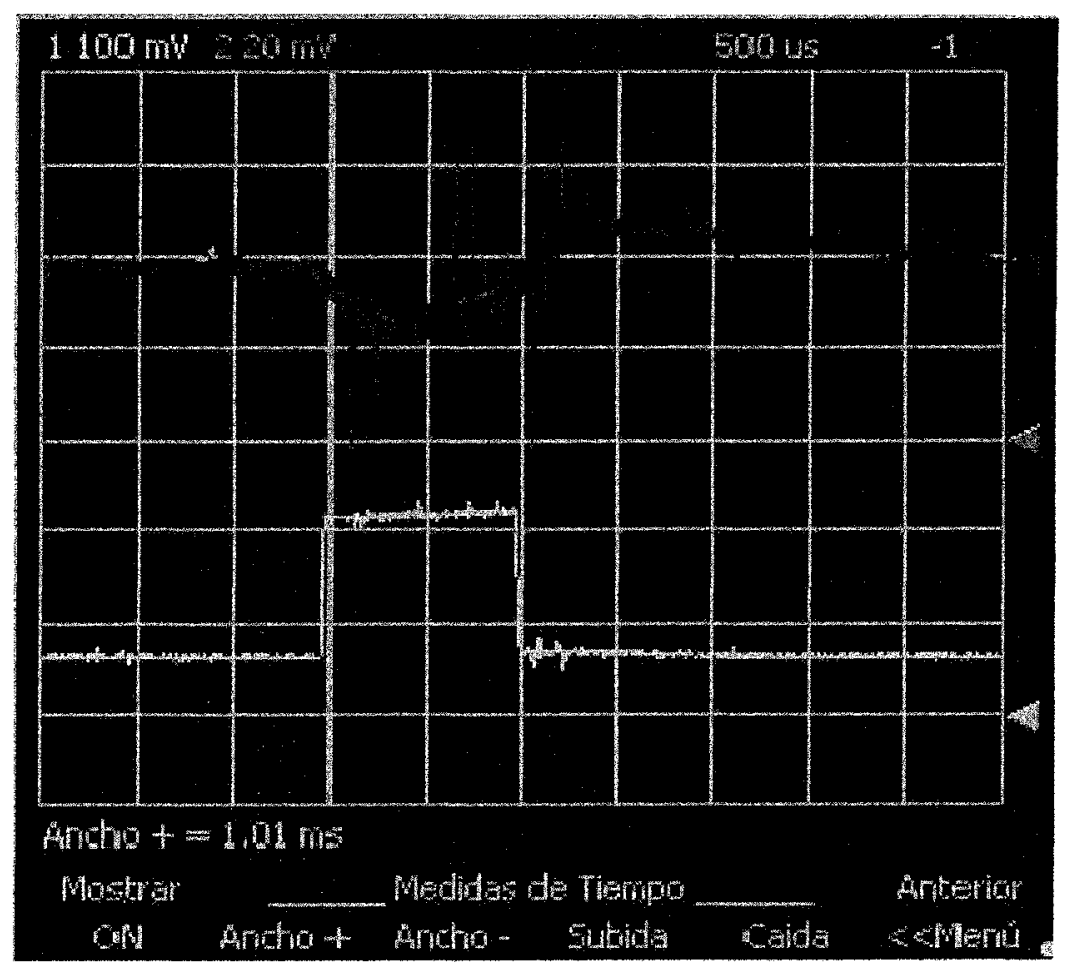

Figure 5. Waveform obtained with a current pulse test

\section{CONCLUSIONS}

The software presented in this article can be used as a complement to the power electronics laboratory lessons, for the study and analysis of DC/DC converters. The developed solution allows, modifying the circuit structure, to analyze the behaviour of the converter in: continuous and/or 
discontinuous operation mode, open and/or close loop. So, the students can obtain the basic parameters that define its answer quality: line regulation, load regulation, input impedance, etc. All it added to the possibility of making the study through a browser increases the versatility and the utility of the system.

In the field of the Power Electronics, the possibility of access of the engineering students to equipment generally of high cost, and therefore of low availability in the educational laboratories, is very important. With this solution it is possible to optimize the use of these equipments in our laboratories.

If you want to know more about the educational laboratory for the study of DC/DC converter, you can visit the website: http://powerlab.dte.uvigo.es and contact with the author: eguizaba@uvigo.es

\section{ACKNOWLEDGMENT}

This research work has been sponsored by Secretaría Xeral de I+D of Xunta de Galicia (Spain) in the project PGIDIT04TIC303002PR. This authors wish to thank the Xunta de Galicia for their financial support.

\section{REFERENCES}

[1] C. Salzmann, D. Gillet, and P. Huguenin, Introduction to real-time control using LabVIEW with an application to distance learning," Int. J. Eng. Eud. vol. 16, pp. 255$272,2000$.

[2] K.K. Tan, T. H. Lee, and F. M. Leu, "Development o a distant laboratory using LabVIEW", Int. J. Eng. Educ. vol. 16, pp. 273-282, 2000.

[3] Kok Kiong, tong Heng, and C. Y. Soh. "Internet-base monitoring of distributed control systems". IEEE Trans. Education. vol. 45 May 2002, page 128-134.

[4] ANSI/IEEE standard 488.2-1992. "IEEE standard codes, Formats, Protocols and Common Commands" The institute of Electrical an Electronics Engineers, Inc, 1988, 1993.

[5] Lisa K. Wells y Jeffrey Travis, "LabVIEW for everyone", Ed. Prentice Hall PTR.

[6] Comer, Douglas E. Y Stevens, David L. "Internetworking with TCP/IP”, Ed. Prentice Hall, 2001.

[7] Jeffrey Travis, "Internet Applications in LabVIEW “, Ed. Prentice Hall PTR. 